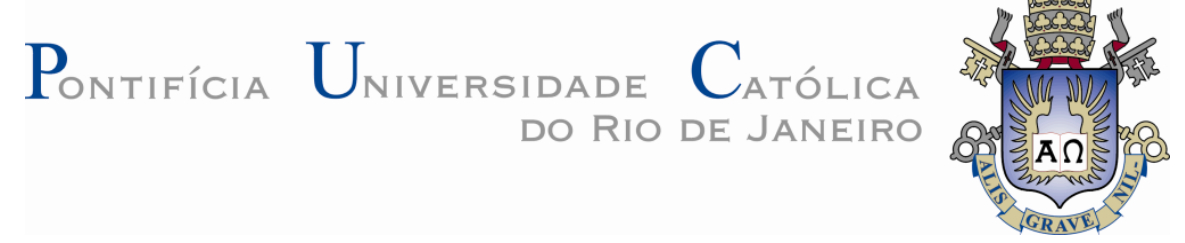

Walace Rosa Laurindo

Estudo experimental de bolhas alongadas no escoamento bifásico horizontal intermitente

Dissertação apresentada ao Programa de Pósgraduação em Engenharia Mecânica da PUC-Rio como requisito parcial para obtenção do título de Mestre em Engenharia Mecânica.

Orientador: Prof. Luis Fernando Alzuguir Azevedo Co-orientador: Dr. Igor Braga de Paula

Rio de Janeiro, Abril de 2012 


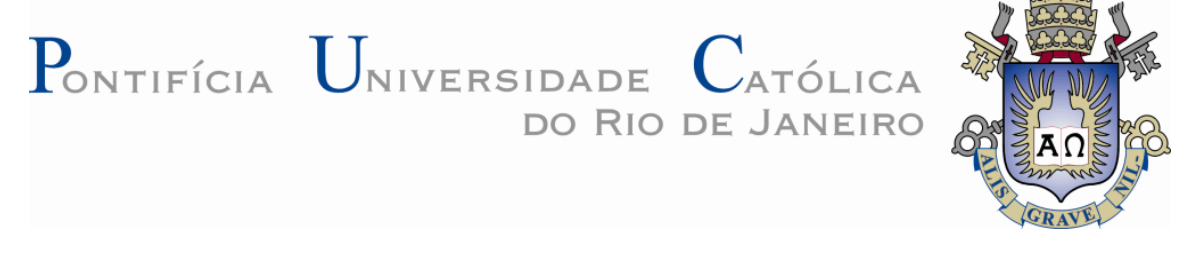

Walace Rosa Laurindo

\title{
Estudo experimental de bolhas alongadas no escoamento bifásico horizontal intermitente
}

Dissertação de Mestrado apresentada como requisito parcial para obtenção do grau de Mestre pelo Programa de Pós-Graduação em Engenharia Mecânica do Departamento de Engenharia Mecânica do Centro Técnico Científico da PUC-Rio. Aprovada pela Comissão Examinadora abaixo assinada.

Prof. Luis Fernando Alzuguir Azevedo

Orientador

Departamento de Engenharia Mecânica - PUC-Rio

\begin{abstract}
Dr. Igor Braga de Paula
Co-orientador

Departamento de Engenharia Mecânica - PUC-Rio
\end{abstract}

Prof. Marco van Hombeeck

Universidade do Estado do Rio de Janeiro - UERJ/IPRJ

Dr. Iberê Nascentes Alves PETROBRAS S. A.

Dr. José Roberto Fagundes Netto PETROBRAS S. A.

Prof. José Eugenio Leal Coordenador Setorial do Centro

Técnico Científico - PUC-Rio

Rio de Janeiro, 04 de abril de 2012 
Todos os direitos reservados. É proibida a reprodução total ou parcial do trabalho sem autorização da universidade, do autor e do orientador.

Walace Rosa Laurindo

Graduou-se com distinção em Engenharia Mecânica na Universidade do Estado do Rio de Janeiro em 2009, com ênfase em petróleo e gás.

Ficha Catalográfica

Laurindo, Walace Rosa

Estudo experimental de bolhas alongadas no escoamento bifásico horizontal intermitente / Walace Rosa Laurindo; orientador: Luis Fernando Alzuguir Azevedo; co-orientador: Igor Braga de Paula - 2012. 161 f. : il.(color.) ; $30 \mathrm{~cm}$

Dissertação (Mestrado) - Pontifícia Universidade Católica do Rio de Janeiro, Departamento de Engenharia Mecânica, 2010.

Inclui bibliografia

1. Engenharia mecânica - Teses. 2. Escoamento bifásico intermitente horizontal. 3. Gás-líquido. 4. Comportamento estatístico. 5. Formato da frente e da traseira da bolha alongada. 6. Processamento de imagens. I. Azevedo, Luis Fernando Alzuguir. II. de Paula, Igor Braga. III. Pontifícia Universidade Católica do Rio de Janeiro. Departamento de Engenharia Mecânica. IV. Título. 
Ao meu amigo Thiago, ao seu filho e esposa, que morreram abraçados na tragédia de janeiro e não puderam me ver mestre. 


\section{Agradecimentos}

Quando o tópico a se escrever em uma dissertação é o agradecimento, é a hora de abrir um belo sorriso e relembrar as pessoas que construíram juntas pequenas "peças" dessa dissertação, cujo quebra-cabeça foi por mim montado ao longo de quase três anos, talvez os mais revolucionários na construção gradativa da minha personalidade. Sempre sonhei em encontrar um local onde o ambiente de trabalho, pesquisa e família, se fundissem em uma só aglomeração. Eis que ao final da graduação meu orientador Marco van Hombeeck, me guiou rumo ao Laboratório de engenharia de fluidos. Lá encontrei alunos de iniciação científica brilhantes, como Giulia, Pedro, Carlos, Bruno, Erick, Renata entre outros... pessoas com quem tive enorme afinidade como Juarez e Leozinho e o super engraçado Léo do SIMDUT e é claro, pessoas e engenheiros brilhantes como Guilherme, Fabão, Paulinha e Helena. Enormes agradecimentos também ao meu co-orientador Igor, o grande mentor de toda a eletrônica e sempre presente nos momentos de leve, médio e grande desespero. Devo muito dessa dissertação a sua enorme dedicação, esforço e rigor. Agradecimentos super especiais ao meu orientador, Luis Fernando, carinhosamente, Lu, o grande pilar dessa família que se tornou o laboratório, onde fui acolhido como sendo "da casa". Agradecimentos não podem faltar aos companheiros de labuta, como Daniel, Marylin e Luciana. Não se pode faltar espaço para os amigos, com perigo a grandes puxões de orelha; aí vão agradecimentos a Ceir, Saymon, Gleison, Johnny, Wallace, Giovane, Darwin e Henrik. Para não levar beliscões das amigas, aí segue os agradecimentos à Jennifer, Luciene, Adriana, Paola, Priscilla e Natali. A todo a minha família, aquele agradecimento, em especial minha tia Cilene e meu tio Ilson, que se esforçaram ao máximo para me dar a mínima estrutura necessária que a CAPES jamais sequer se preocupou em perguntar. Se desejamos um país na vanguarda da tecnologia, devemos nos preocupar em dar estrutura, incentivos e suporte ao grande patrimônio desse 
país que são os alunos. Agradeço a minha mãe, Maria das Graças, ao meu padrasto, muito mais que um pai, Valney, e aos irmãos Willian e Alan. Agradeço a minha avó, Dorcelina Rosa, pela convivência e por resistir aos sustos que me deu ao longo dessa dissertação. Ela foi brava, valente, e suportou tudo para me ver escrever isso aqui. Agradecimentos a teoria da existência divina. Pude sinceramente perceber que enquanto caminhava, muitas das peças do tabuleiro eram mexidas por alguém com extrema sabedoria. Por último, agradeço a você, que teve paciência para ler duas páginas de agradecimento. Siga em frente rumo a leitura minuciosa da dissertação. Foi para isso que ela foi cuidadosamente escrita e que a leia, com no mínimo metade da alegria com que a escrevi. 


\section{Resumo}

Laurindo, Walace Rosa, Azevedo, Luis Fernando Alzuguir. Estudo experimental de bolhas alongadas no escoamento bifásico horizontal intermitente. Rio de Janeiro, 2012. 161p. Dissertação de Mestrado Departamento de Engenharia Mecânica, Pontifícia Universidade Católica do Rio de Janeiro.

Ao longo deste trabalho, diversas características importantes para a dinâmica do escoamento intermitente foram analisadas experimentalmente. Todos os testes foram realizados numa tubulação com 0,0508 $\mathrm{m}$ de diâmetro interno, em configuração horizontal, utilizando água e ar e abrangendo uma região onde o escoamento passa por uma complexa transição do regime de "bolha alongada", caracterizado por longas bolhas de gás localizadas próximo ao topo da tubulação, para o regime "golfada", caracterizado por uma intensa quantidade de bolhas dispersas de gás e bolhas alongadas com formatos distorcidos e frentes mais distantes do topo da tubulação. Nesta região verifica-se uma notável carência de técnicas experimentais capazes de extrair informações quantitativas dos fenômenos característicos desses regimes. Através de um sistema de interruptores de feixe de infravermelho, o comportamento estatístico dos comprimentos dos pistões de líquido, das frequências de passagem dos pistões e do comprimento da bolha alongada puderam ser investigados. Uma extensa comparação dos dados com trabalhos disponíveis na literatura foi realizada, validando os resultados experimentais obtidos. Em conjunto, foi utilizada a técnica óptica, não intrusiva, conhecida como técnica de sombra (shadow technique), combinada com uma câmera digital de alta taxa de aquisição de imagens. Um procedimento inovador de processamento das imagens foi desenvolvido, permitindo a extração de informações quantitativas de regimes complexos com intensa quantidade de bolhas dispersas. Resultados importantes como o abaixamento do nariz da bolha como uma função da competição entre as forças de gravidade e de inércia puderam ser verificados e confirmados quantitativamente pela primeira vez.

\section{Palavras-chave}

Escoamento bifásico intermitente horizontal; gás-líquido; comportamento estatístico; formato da frente e da traseira da bolha alongada; processamento de imagens. 


\section{Abstract}

Laurindo, Walace Rosa, Azevedo, Luis Fernando Alzuguir (Advisor). Experimental study of elongated bubbles in horizontal two-phase intermittent flow. Rio de Janeiro, 2012. 161p. MSc. Dissertation Departamento de Engenharia Mecânica, Pontifícia Universidade Católica do Rio de Janeiro.

The present work experimentally studied several relevant dynamic characteristics of gas-liquid intermittent flow through a horizontal pipe. All tests were performed in a test section formed by $50.8 \mathrm{~mm}$ internal diameter pipe using air and water as working fluids. The tests covered a region where the flow undergoes a complex transition from "elongated bubble flow" regime, characterized by long gas bubbles located near the top of the pipe, to the "slug flow" regime, characterized by a large amount of dispersed gas bubbles in the liquid slug and elongated bubbles with distorted shapes and fronts displaced from the top of the pipe. In this region there is a notable lack of experimental techniques capable of extracting quantitative information of the characteristic dynamic phenomena involved. A set of infrared optical switches was employed to obtain statistical information on liquid piston lengths and frequency, as well as the elongated bubble lengths. An extensive comparison of the data obtained with works available in the literature was performed, validating the experimental results and the technique employed. Also, a high frame rate camera with back illumination was employed to capture images of the nose and tail of gas bubbles. An innovative procedure for image processing was developed, allowing for the extraction of original quantitative information on the complex shapes of the bubble and its relationship with flow variables. Relevant information such as the lowering of the bubble nose as a result of competing gravitational and inertial forces could be verified and confirmed quanti tatively seemingly for the first time.

\section{Keywords}

Two-phase intermittent slug flow; gas-liquid; statistics behavior; shape of the front and rear of elongated bubbles; image processing. 


\section{Sumário}

1 Introdução 15

1.1. Organização do trabalho 20

2 Revisão bibliográfica $\quad 21$

2.1. Conceitos e fundamentos básicos 21

2.2. Uma breve discussão sobre a modelagem do escoamento Intermitente e o problema de fechamento 25

2.3. Formação e desenvolvimento do escoamento intermitente 27

2.3.1. Teoria não viscosa de Kelvin-Helmholtz 28

2.3.2. Teoria de instabilidade do comprimento de uma grande onda viscosa $\quad 29$

2.3.3. Teoria de estabilidade dos pistões $\quad 29$

2.3.4. Desenvolvimento 31

2.4. Velocidade média de translação da bolha alongada 33

2.5. A importância do estudo do formato da frente e da traseira da alongada $\quad 39$

2.6. Comprimento do pistão de líquido $\quad 44$

2.7. Frequência de passagem dos pistões de líquido 47

2.8. Comprimento da bolha alongada 50

3 Montagem experimental $\quad 52$

3.1. Descrição geral da montagem experimental 52

3.2. Sistema de escoamento de água 56

3.3. Sistema de escoamento de ar 56

3.4. Sistema de separação $\quad 57$

3.5. Estação de medição 58

3.5.1. Sistema de interruptores de feixe 59

3.5.2. Caixa de visualização $\quad 61$

3.5.3. Painel de LED's 61

.5.4. Câmera 62

4 Procedimento experimental e redução de dados 64

4.1. Operação da seção de testes 64 
4.2. Sistema de interruptores de feixe

4.2.1. Cálculo dos parâmetros de interesse 68

4.3. Algumas definições básicas de estatística 71

4.3.1. Média $\quad 71$

$\begin{array}{ll}\text { 4.3.2. Medidas de variação } & 71\end{array}$

4.3.3. Intervalos de confiança $\quad 72$

4.3.4. Critério de convergência para o tamanho das amostras 76

4.3.5. Desvio médio absoluto 76

5 Descrição da técnica de medição e processamento de imagens 78

5.1. Técnica de sombra $\quad 78$

5.2. Configuração experimental da técnica 79

5.3. Processamento de imagens $\quad 81$

5.3.1. Processamento de imagens da frente da bolha alongada 82

5.3.2. Processamento de imagens da traseira da bolha alongada 89

6 Resultados $\quad 92$

6.1. Matriz de testes 92

6.2. Comparação entre os métodos de medição utilizados 96

6.3. Comprimento do pistão de líquido 98

6.4. Frequência de passagem dos pistões de líquido 105

6.5. Comprimento da bolha alongada 109

6.6. Velocidade de translação da frente da bolha 112

6.7. Análise da mudança do formato do nariz e da traseira da bolha 119

6.7.1. Análise da mudança do formato do nariz da bolha 119

6.7.1. Análise da mudança do formato da traseira da bolha 125

$\begin{array}{ll}7 \text { Conclusões } & 130\end{array}$

8 Bibliografia $\quad 134$

$\begin{array}{ll}\text { Apêndice } & 141\end{array}$

$\begin{array}{ll}\text { A Metodologia para a avaliação de incertezas } & 141\end{array}$

A.1. Avaliação das incertezas nas varáveis medidas pelo sensor 141

A.2. Tabelas com as estimativas das incertezas das variáveis

medidas pelo sensor 
A.3. Histogramas do comprimento do pistão de líquido, da frequência de passagem dos pistões e comprimento da bolha alongada

A.4. Avaliação das incertezas nas variáveis medidas pelo

processamento de imagens 


\section{Lista de Variáveis}

\section{Letras Latinas}

$a, b, c$ - constantes da solução de Benjamin

$a_{1}, a_{2}, a_{3}$ - constantes da correlação de Fossa

$A$ - área da seção transversal da tubulação

$c^{\prime}$ - fator de calibração

$C, C^{\prime}, C_{\infty}$ - coeficiente relacionado à contribuição da velocidade de mistura, velocidade de translação da onda, parâmetro adimensional

$d$ - distância

$D$ - diâmetro interno da tubulação

Eo - número de Eötvos

$f$ - frequência média de passagem

Fr - número de Froude

$g, g^{\prime}$ - aceleração da gravidade, aceleração da gravidade modificada

$h, h^{\prime}, \hat{h}$ - altura média do filme de líquido , altura instantânea do filme de líquido, amplitude da onda

$i, i^{\prime}$ - contador do número de fases, número imaginário

$j$ - velocidade superficial

$k, k^{\prime}$ - contador do somatório, número de onda

$L$ - comprimento

$n$ - número total de dados experimentais

$N$ - tamanho da amostra

$Q$ - vazão volumétrica

$R, R^{\prime}$ - coeficiente de determinação, coordenada de linha de corrente na solução de Benjamin

Re - número de Reynolds

$s$ - desvio padrão

$S$ - escorregamento

St - número de Strouhal

$t, t^{\prime}$ - tempo, parâmetro da distribuição de $t$-de Student

$u$ - velocidade média 
$x$ - coordenada axial

$y$ - coordenada transversal

\section{Letras gregas}

$\alpha$ - fração volumétrica

$\lambda, \lambda^{\prime}$ - fração de volume (holdup), comprimento de onda

$\pi$ - número $p i$

$\theta$ - inclinação

$\mu$ - viscosidade

$\rho$ - massa específica

$\sigma$ - tensão superficial

$\Sigma$ - parâmetro de tensão superficial

$\Delta$ - variação

$\Gamma$ - número de viscosidade inverso

$\chi$ - variável qualquer

\section{Subscritos}

$b$ - bolhas dispersas

$B$ - bolha alongada

correl - correlação

crit - crítico (a)

$d$ - deslizamento

ent - entrada

exp - experimental

$f$ - filme de líquido

$G$ - fase gasosa

$L$ - fase líquida

$m$ - mistura

max - máximo (a)

$S$ - pistão de líquido

sai - saída

$T$ - traseira da bolha alongada

$u$ - célula unitária 


\section{Siglas}

CMOS - Complementary Metal-Oxide-Semiconductor

$C V$ - Coeficiente de Variação

$D M A$ - Desvio Médio Absoluto

FEP - Fluorinated Ethylene Propylene

LED - Light Emitting Diode

VOF - Volume of Fluid 